\title{
A systematic comparison of the MetaCyc and KEGG pathway databases
}

\author{
Tomer Altman, Michael Travers, Anamika Kothari, Ron Caspi and Peter D Karp*
}

\begin{abstract}
Background: The MetaCyc and KEGG projects have developed large metabolic pathway databases that are used for a variety of applications including genome analysis and metabolic engineering. We present a comparison of the compound, reaction, and pathway content of MetaCyc version 16.0 and a KEGG version downloaded on Feb-27-2012 to increase understanding of their relative sizes, their degree of overlap, and their scope. To assess their overlap, we must know the correspondences between compounds, reactions, and pathways in MetaCyc, and those in KEGG. We devoted significant effort to computational and manual matching of these entities, and we evaluated the accuracy of the correspondences.
\end{abstract}

Results: KEGG contains 179 module pathways versus 1,846 base pathways in MetaCyc; KEGG contains 237 map pathways versus 296 super pathways in MetaCyc. KEGG pathways contain 3.3 times as many reactions on average as do MetaCyc pathways, and the databases employ different conceptualizations of metabolic pathways. KEGG contains 8,692 reactions versus 10,262 for MetaCyc. 6,174 KEGG reactions are components of KEGG pathways versus 6,348 for MetaCyc. KEGG contains 16,586 compounds versus 11,991 for MetaCyc. 6,912 KEGG compounds act as substrates in KEGG reactions versus 8,891 for MetaCyc. MetaCyc contains a broader set of database attributes than does KEGG, such as relationships from a compound to enzymes that it regulates, identification of spontaneous reactions, and the expected taxonomic range of metabolic pathways. MetaCyc contains many pathways not found in KEGG, from plants, fungi, metazoa, and actinobacteria; KEGG contains pathways not found in MetaCyc, for xenobiotic degradation, glycan metabolism, and metabolism of terpenoids and polyketides. MetaCyc contains fewer unbalanced reactions, which facilitates metabolic modeling such as using flux-balance analysis. MetaCyc includes generic reactions that may be instantiated computationally.

Conclusions: KEGG contains significantly more compounds than does MetaCyc, whereas MetaCyc contains significantly more reactions and pathways than does KEGG, in particular KEGG modules are quite incomplete. The number of reactions occurring in pathways in the two DBs are quite similar.

Keywords: Pathway databases, Database comparison

\section{Background}

MetaCyc [1-8] and KEGG [9-15] are large metabolic pathway database (DB) projects that have been under development for more than a decade. Both projects provide reference pathways that are used to predict the metabolic pathways present in an organism from the annotated genome of that organism. MetaCyc has been utilized for pathway prediction in the BioCyc database collection [2], and in many other Pathway/Genome Databases developed by researchers around the world [16]. The KEGG

*Correspondence: pkarp@ai.sri.com

Bioinformatics Research Group, SRI International, Menlo Park, USA project consists of both a reference pathway database, and the resulting projection of the reference pathways onto organisms with sequenced genomes. KEGG is also widely used.

The goal of this article is to compare the data content of MetaCyc with the data content of the KEGG reference pathway DB to provide an understanding of their relative sizes, their degree of overlap, their scope, and the breadth of data that they provide. These questions are particularly important because the accuracy of metabolic pathway prediction is directly dependent upon the coverage of the reference pathway $\mathrm{DB}$ that the pathway predictor 
utilizes [17]. Most metabolic pathway predictors predict the presence of pathways that exist in a reference pathway DB. Another important and active area of research is the development of steady-state metabolic flux models. These models are highly dependent upon the source of metabolic reactions from which the models are derived. Our analysis considers KEGG and MetaCyc because they are the largest curated databases of metabolic reactions and pathways (see Table 2 in [18]), containing significantly more reactions than Rhea, BiGG, UniPathway, BioPath, and Reactome. Although The SEED and BRENDA contain comparable numbers of reactions, the metabolic content of The SEED is largely derived from KEGG [19], and BRENDA does not include metabolic pathways.

Metabolic pathway data can be thought of as consisting of three tiers: the metabolites form the lowest tier; reactions are built upon metabolites, and pathways are built upon reactions. Our analysis considers all three tiers. Our comparison does not include other aspects of KEGG or BioCyc, such as their orthology data, or their genomebased pathway predictions for sequenced organisms.

To analyze the degree of overlap of MetaCyc and KEGG, we must know the correspondences between compounds, reactions, and pathways in MetaCyc, and those in KEGG. Establishing these correspondences is a non-trivial problem because of the non-standard terminologies used in the scientific literature for these three entities (e.g., large numbers of synonyms are used for a given chemical compound), because some metabolites lack chemical structures in one DB or the other, and because stereochemical information is not present for every metabolite that contains a stereo center, thus precluding complete matching using chemical structures. We devoted significant effort to the problem of matching metabolites and reactions (see Methods). As a result, many MetaCyc compounds and reactions now contain DB links to the corresponding objects in KEGG. MetaCyc pathways do not contain such links because metabolic pathways have more subjective definitions than do metabolites and reactions, and hence we do not expect there to be exact correspondences between the pathways in MetaCyc and KEGG.

Consider the following problem that is common to this study, to other recent studies that compare bioinformatics DBs [20,21], and to efforts that integrate data from multiple metabolic DBs [18,22-25]. To compare, for example, the metabolite complements of two pathway DBs, we must know which metabolites within the DBs correspond to one another. But the algorithms that compute such correspondences are imperfect. If metabolite $M$ in one $\mathrm{DB}$ is found to have no counterpart in the other $\mathrm{DB}$, does this observation reflect the true state of affairs, or a false-negative result by the matching algorithm? We present a method for approaching this problem - namely, to sample matched and unmatched objects from the DBs and manually validate or search for correspondences to quantify the accuracy of the correspondences. A caveat for all of our analyses is that they are dependent on the accuracy and completeness of the compound and reaction correspondences that we have curated, imported, and computed. All curated, imported, and inferred links from MetaCyc compounds and reactions to their corresponding entries in KEGG may be found in the MetaCyc files that are available for download from the web.

We also introduce a general method for assessing where is the semantic overlap between two DBs: we use enrichment/depletion analysis to detect whether areas of one DB are disproportionately populated or depopulated with respect to the other $\mathrm{DB}$.

\section{Methods}

We used MetaCyc version 16.0 (released on February 17, 2012) and a version of KEGG downloaded on February 27, 2012 for the purpose of this study, unless otherwise noted. The KEGG datasets were downloaded using the KEGG SOAP object-retrieval web services [26] using the following functions via the BioBike software [27]: list_organisms, binfo, list_pathways, bfind, bget, get_compounds_by_pathway, get reactions_by_pathway, and get_element_relations_by_pathway.

The KEGG data were loaded into a new Pathway Tools [28] DB ("KeggCyc") that uses the same schema as MetaCyc. The loaded KEGG datasets were COMPOUND, REACTION, MAP, and MODULE. KEGG chemical structures were obtained from the COMPOUND dataset, not from KEGG MOL files. This KEGG loader was implemented in Common Lisp, and is available in the Additional file 1. Once loaded into a Pathway Tools DB, the KEGG data can be queried and visualized using Pathway Tools. The KEGG analyses reported herein were performed using Common Lisp programs that queried KeggCyc.

\section{Comparing compound data}

Correspondences between MetaCyc compounds and KEGG compounds are encoded as MetaCyc compounds DB links to the corresponding KEGG compound. Such DB links are added to MetaCyc by several means. The MetaCyc curation staff members add such links during their manual curation. In addition, we submit MetaCyc compounds with chemical structures to the PubChem standardization pipeline in order to match MetaCyc compound structures with PubChem Compound entries. KEGG compounds are also periodically processed by the same PubChem standardization pipeline. We have imported links to KEGG compounds from PubChem Compound dataset that are linked to both MetaCyc and 
KEGG compounds. We also received compound correspondences between MetaCyc and KEGG compounds from other research efforts, which we checked for errors before programmatically importing them into MetaCyc (John Bates, Dylan Chivan, personal communication).

We implemented a rule-based system to predict additional compound matches between MetaCyc and KEGG. We defined a set of compound match features, such that a proposed compound match required one or more features from the set, along with a compound name exact string match, in order for a match to be predicted. We defined the set of compound features for MetaCyc and KEGG compounds as molecular fingerprint matching via PubChem [29] coupled with the Pathway Tools compound structure matcher, exact stereo-structure matching, and "all-but-one" inference. We utilized the pre-computed molecular fingerprints for KEGG and MetaCyc compounds in PubChem to detect compound pairs that had a Tanimoto coefficient [30] greater than 0.75 . We then further filtered the compound pairs by using the compound structure matcher from Pathway Tools, which can detect compounds with the same structure, even if there are differences in protonation state.

"All-but-one" compound match inference is where a pair of known corresponding reactions, one from MetaCyc and the other from KEGG, have all of their substrates matched except for one pair of substrates $-C_{M}$ from the MetaCyc reaction and $C_{K}$ from the KEGG reaction. If $C_{M}$ does not already have a compound link to a KEGG compound, and $C_{K}$ does not already have a compound link to a MetaCyc compound, we infer a match between $C_{M}$ and $C_{K}$.

For the exact string matching of the compound common name, name strings were "canonicalized" to remove differences such as punctuation and capitalization prior to checking for exact matches. Any pair of compounds consisting of a MetaCyc compound and a KEGG compound that had at least one feature match from the set, had an exact string match of their names, and had no contradictory matches (e.g., where our rule-based system inferred that a single MetaCyc compound had two matching KEGG compounds) were inferred as compound matches. Automatically inferred compound matches were randomly sampled for review by our curation staff for quality assurance. 1214 compound correspondences were inferred using this system.

The International Union of Pure and Applied Chemistry International Chemical Identifier (InChI) is a method of generating a unique string representation of a chemical compound structure [31]. We used the official InChI software package (version 1.02) for generating standard InChI strings from compounds with structures in MetaCyc and KEGG. The InChI executable was called with the following arguments: -STDIO -NoLabels -AuxNone. We infer two compounds as having matching structure if their standard InChI strings are identical. We used InChI strings to detect compound matches between MetaCyc and KEGG, and to detect duplicate compounds within MetaCyc or KEGG. InChI strings may be used to detect differences in stereo-center orientation between two compounds that otherwise have the same structure. Both KEGG and MetaCyc contain compounds with one or more unspecified stereo centers, and thus using InChI strings to detect matching compounds may miss some legitimate matches. Furthermore, InChI strings are different for two different protonation states [1] of the same compound, and thus equivalent compounds with different protonation states between KEGG and MetaCyc may be overlooked by comparing InChI strings. For related work on establishing correspondences between metabolic databases using string matching, see MetRxn [23] (which matches metabolites on a canonical SMILES structure representation) and BKM-react [24] (which matches metabolites on InChI string and name).

We assessed the accuracy of our correspondences between KEGG and MetaCyc compounds. Specifically, we applied the binomial distribution to estimate the proportion of false negatives at a confidence level of $90 \%$ and with a confidence interval of $10 \%$, which indicated that 68 samples were necessary. Thus, we sampled 68 MetaCyc compounds with no inferred link to a compound in KEGG and manually searched for a corresponding KEGG compound. We found 9 MetaCyc compounds (13.2\%) that did have a corresponding entry in KEGG. This result implies that we have $90 \%$ confidence that the true proportion of false negative predictions is between 0.0013 and 0.232 , meaning that as many as 1650 MetaCyc compounds remain to have their corresponding KEGG compound determined. We used a separate sampling of 68 MetaCyc compounds with inferred links to KEGG compounds in order to estimate the proportion of false positives at the same confidence level and interval. Manual verification of the correspondences identified one compound (1.5\%) that had an incorrect correspondence. This implies that we have $90 \%$ confidence that the true proportion of false positive predictions is between $8.4 \times 10^{-4}$ and $11.5 \%$, or as many as 140 compounds with incorrect correspondences. With the false positive and false negative rates estimated, the overall accuracy is $85.3 \%$, with an upper bound of $99.9 \%$ and a lower bound of $65.3 \%$ for the given confidence level and interval.

In addition to reporting the number of compounds in the intersection between MetaCyc and KEGG for various compound categories, we compute the Jaccard coefficient as a measure of the similarity between the two sets. The Jaccard coefficient can be simply defined as the size of the intersection of the two sets divided by the size of the union of the two sets [30]. Thus, two sets that are identical 
will have a Jaccard coefficient of one, while two disjoint sets will have a Jaccard coefficient of zero. We use the Jaccard coefficient in comparing the overlap of reactions in MetaCyc and KEGG as well.

\section{Comparing reaction data}

The KEGG COMPOUND dataset contains entries that are pharmaceuticals or glycan compounds, and thus have matching entries in the KEGG DRUG and GLYCAN datasets, respectively. These objects are designated with "D" and "G" prefixes in their identifiers and are duplicates of the standard compound objects, in the COMPOUND dataset, that use an identifier prefix of " $\mathrm{C}$ ". We found that the KEGG REACTION dataset contains duplicate reactions, where one version of a reaction will use the " $C$ " identifiers, while the other version of the same reaction would use the "G" or "D" identifiers. We analyzed only reactions that consisted of " $\mathrm{C}$ " identifiers for the purpose of this study to avoid double-counting reactions.

We used a combination of manual curation, a computational rule-based system for inferring reaction correspondences, and bulk extraction of reaction correspondences from databases such as BKM-React, GO, MetRxn, and Rhea $[23,24,32,33]$ to create links between MetaCyc and KEGG reactions.

A proposed reaction match required a significant similarity of reactants and products, along with at least one match from a set of three reaction features. The first feature from the set checked the enzymes that catalyzed the pair of reactions from MetaCyc and KEGG to see if they had at least one UniProt [34] accession number in common. The second feature employed exact matches of International Union of Biochemistry and Molecular Biology (IUBMB) Enzyme Commission (EC) [35] enzyme classification number matches. The third feature checked for exact enzymatic activity name matches, using KEGG REACTION entry names and enzymatic activity names from MetaCyc enzyme information associated with reaction objects. Reaction and enzymatic activity name strings were "canonicalized" to remove differences such as punctuation and capitalization prior to checking for exact matches.

To detect similarities of reaction reactants and products, we defined a feature by representing reactions as column vectors in a stoichiometric matrix [36]. In brief, a stoichiometric matrix represents reactions as columns, and each distinct compound in the metabolic network is represented by a row. Matrix values are zero unless a particular compound participates in a particular reaction, in which case the coefficient of the compound in the reaction is inserted at the corresponding row and column in the matrix. Reactant coefficients are entered into the matrix as negative values; product coefficients are positive.
We inferred a match if the absolute value of the cosine similarity [30] of the MetaCyc stoichiometry vector and the KEGG stoichiometry vector was greater than 0.6 (with identical reactions having a cosine similarity of 1.0), along with the reaction pair having one or more matches from the set of three reaction match features, and no contradictory matches. Finally, any remaining pairs of reactions between KEGG and MetaCyc that had no other matches, yet had the same EC number, and the EC number based matching was one-to-one, were inferred as matches. 3211 reaction object correspondences were inferred using this system.

We sought to assess the accuracy of our reaction correspondences using a sampling procedure. As described for compounds, we sampled 68 MetaCyc reactions with no inferred link to a reaction in KEGG and 68 MetaCyc reactions with an inferred link to a reaction in KEGG. We found 9 MetaCyc reactions without links (13.2\%) that did have a corresponding entry in KEGG. This implies that we have $90 \%$ confidence that the true proportion of false negative predictions is between 0.0013 and 0.232 , or as many as 1636 MetaCyc reactions remaining to have their corresponding KEGG reaction determined. We found 6 reactions $(8.8 \%)$ that had an incorrect link. This implies that we have $90 \%$ confidence that the true proportion of false positive predictions is between .0018 and 0.188 , or as many as 603 reactions with incorrect links. With the false positive and false negative rates estimated, the overall accuracy is $78.0 \%$, with an upper bound of $99.69 \%$ and a lower bound of $58 \%$ for the given confidence level and interval.

To assess the balance state of reactions in MetaCyc and KEGG we used the reaction balance checker in Pathway Tools. This software counts the atoms present on both sides of a given reaction and checks for equality of atom counts. Although MetaCyc compounds are protonated consistently relative to a defined $\mathrm{pH}$ [37], we are not aware of any consistent protonation among KEGG compounds. To avoid unduly penalizing KEGG reactions, we also checked the balance state of reactions in both MetaCyc and KEGG for all atoms aside from hydrogen.

\section{Comparing pathway data}

MetaCyc contains two types of pathways: base pathways are individual metabolic pathways (example: TRPSYN-PWY, "tryptophan biosynthesis"); super pathways combine sets of base pathways, super pathways, and individual reactions into larger composite pathways (example: ALL-CHORISMATE-PWY, "superpathway of chorismate metabolism"). Similarly, KEGG contains analogous notions of modules and maps. KEGG modules were introduced to "define tighter functional units than KEGG PATHWAY" [38]. The KEGG pathway data were obtained from the MODULE and MAP datasets. 
KEGG modules are of four types: pathway modules, structural protein and RNA complexes, functional sets, and signature modules. The latter three types of modules are not metabolic pathways and are excluded from our analyses, thus excluding more than half of KEGG modules. KEGG defines three "Global Pathways" in the MAP dataset: map01100 ("Metabolic Pathways"), map01120 ("Microbial metabolism in diverse environments"), and map01110 ("Biosynthesis of secondary metabolites"). These pathways include thousands of reactions from many other maps. Since these three large maps are qualitatively different entities than the other KEGG maps, we excluded them from the analysis herein. Furthermore, KEGG pathway classes where none of the associated pathway instances have any metabolic reaction data (such as the "Environmental information processing" pathway class) were also excluded since they do not contain metabolic pathway data.

\section{Results}

\section{Compound comparison}

Table 1 compares the number of chemical compounds found in MetaCyc and KEGG. Table 2 compares the types of compound data present in KEGG and MetaCyc, and the degree to which different data fields are populated in the two DBs.

Table 3 compares additional MetaCyc and KEGG compound object attributes. The table omits KEGG compound attributes called ENZYME and PATHWAY, which represent relationships between KEGG objects. The equivalent relationships can easily be extracted from MetaCyc via other objects and attributes, and thus are not represented as direct attributes of the compounds.

\section{Reaction comparison}

Table 4 compares the number of reactions between MetaCyc and KEGG. Tables 5 and 6 compare different aspects of reaction attributes between MetaCyc and KEGG. Note that KEGG provides its data in a number of other formats. For example, KEGG KGML files contain information on reaction reversibility, but because our study is limited to data accessible via the SOAP web services, that reversibility information is not listed in Table 6.
Table 2 Comparison of compound data content in MetaCyc and KEGG

\begin{tabular}{lcc}
\hline & MetaCyc & KEGG \\
\hline Compounds & 11991 & 15161 \\
Compounds with structures & 10546 & 14621 \\
Compounds with comments & 1486 & 2997 \\
Mean comment length & 47.69 & 6.51 \\
Mean names per compound & 2.37 & 1.62 \\
Mean DB links per compound & 1.71 & 3.71 \\
Mean associated reactions & 3.59 & 2.17 \\
Mean associated pathways (all) per compound & 1.78 & 0.67 \\
Duplicate compounds & 36 & 251
\end{tabular}

Compound entries in either DB may not have information on their chemical structures, and may not have comments describing the properties of the compound. Associated pathways of a compound include base pathways and superpathways in MetaCyc and KEGG maps and modules. Compounds were considered duplicates if they had identical standard InChl strings.

Since hydrogen imbalance in KEGG reactions can be the result of inconsistent protonation states of the compounds involved, we consider such imbalance as a potentially less serious problem than imbalance by other elements. Thus, we conducted a special set of analyses for reaction balancing where we did not count hydrogen atoms.

Two reactions $R_{1}$ and $R_{2}$ are duplicates if the reactants of $R_{1}$ are the same as the reactants of $R_{2}$, and the products of $R_{1}$ are the same as the products of $R_{2}$, or ditto for the reverse of $R_{1}$.

\section{Pathway comparison}

A summary of MetaCyc base and super pathways, and KEGG modules and maps is presented in Table 7. A comparison of pathway data in MetaCyc and KEGG is presented in Table 8.

A histogram plot of the frequency of MetaCyc base pathway sizes (by reaction count) and KEGG modules sizes (by reaction count) is presented in Figure 1, and a histogram plot of the frequency of MetaCyc super pathways and KEGG maps sizes by reaction count is presented in Figure 2.

Table 1 Comparison of chemical compounds in MetaCyc and KEGG

\begin{tabular}{|c|c|c|c|c|c|c|c|}
\hline Category & M(all) & M(base) & $M($ super) & $\mathrm{K}(\mathrm{all})$ & K(module) & $\mathrm{K}(\mathrm{map})$ & Common \\
\hline All chemical compounds & 11991 & & & 15161 & & & $5120(0.23)$ \\
\hline All reaction substrates & 8891 & & & 6912 & & & $4232(0.37)$ \\
\hline Pathway reaction substrates & 5523 & 5371 & 5523 & 4759 & 828 & 4759 & $2384(0.30)$ \\
\hline
\end{tabular}

For each type of compound (row), we report the number of compounds in MetaCyc, the number of compounds in KEGG, and the number of compounds in common between MetaCyc and KEGG. "All chemical compounds" includes both compound classes and compound instances for MetaCyc; for KEGG it includes all compounds in the KEGG COMPOUND file. "All reaction substrates" is the union of all literal reaction substrates (reactants plus products) in the specified DB. M(all): all MetaCyc compounds; $\mathrm{M}$ (base): compounds in MetaCyc base pathways; M(super): compounds in MetaCyc super pathways; K(all): all KEGG compounds; K(module): compounds in KEGG module; K(map): compounds in KEGG map; Common: corresponding compounds by total number and by the Jaccard coefficient in parentheses. 
Table 3 A comparison of MetaCyc and KEGG compound attributes, for those attributes where one hundred or more objects have a value for that attribute

\begin{tabular}{lclr}
\hline MetaCyc & KEGG & \\
Attribute & Frequency & Attribute & Frequency \\
\hline Monoisotopic-MW & 9475 & Exact_Mass & 14611 \\
Molecular-Weight & 9431 & Mol_Weight & 14611 \\
\hline Creation-date & 11705 & & \\
Creator & 10573 & & \\
SMILES & 10546 & & \\
InChl & 9222 & \\
Regulates & 3573 & \\
Credits & 2895 & \\
Gibbs-0 & 1033 & \\
Cofactors-Of & 563 &
\end{tabular}

The table presents shared attributes (note that the name for the same conceptual attribute may differ between MetaCyc and KEGG), and attributes unique to MetaCyc; the two attribute sets are further sorted based on the number of objects containing non-null values for each attribute. Gibbs- 0 is the Gibbs free energy of formation of a compound. Creator, Creation-Date, and Credits provide data provenance. KEGG compound attribute data are derived from the KEGG COMPOUND dataset.

See Table 9 for a comparison of other MetaCyc and KEGG pathway object attributes. Note that the KEGG module attribute called COMPOUND links compound objects with modules. In MetaCyc the equivalent relationship is represented via reaction objects, and thus there is no need for an additional attribute.

We next sought to understand how much KEGG and MetaCyc pathways overlap, a question that we approach from several directions. Table 10 explores the degree to which KEGG and MetaCyc pathways cover their respective reaction spaces. For example, if a KEGG module $M$ contains six reactions, and none of those reactions are present in (linked to) MetaCyc (based on the MetaCyc reaction links to KEGG reactions), we think of $M$ as unique to KEGG because it covers a part of reaction space that is not present in MetaCyc. We say that a pathway class $C$ is enriched/depleted for reaction links if the reactions $R_{i}$ contained within pathway instances $P_{j}$ in $C$, are enriched/depleted (as described in Section 'Pathway comparison') for links to reactions in KEGG. Such analysis is, of course, limited by the fact that our reaction links are imperfect. Table 10 summarizes the fraction of MetaCyc pathways for which all, some, or none of their reactions
Table 5 Comparison of reaction data content in MetaCyc and KEGG

\begin{tabular}{lcc}
\hline Category & MetaCyc & KEGG \\
\hline Reaction instances & 10262 & 8879 \\
Duplicate reactions & 279 & 341 \\
Reactions with comments & 3206 & 3022 \\
Unbalanced reactions (not counting hydrogen) & 474 & 872 \\
Unbalanced reactions (counting hydrogen) & 532 & 1475 \\
Mean associated pathways & 0.84 & 0.90
\end{tabular}

We report the number of reactions, the number of duplicate reactions, the number of reactions with comments, the number of unbalanced reactions disregarding hydrogen imbalance, the number of unbalanced reactions including hydrogen imbalance, and the average number of associated pathways. Associated pathways of a reaction include base pathways and superpathways in MetaCyc and KEGG modules and maps.

correspond to reactions in KEGG, and the converse the fraction of KEGG pathways for which all, some, or none of their reactions correspond to reactions in MetaCyc. MetaCyc pathways include a total of 3,901 reactions not found in KEGG, whereas KEGG pathways include a total of 3,852 reactions not found in MetaCyc.

Another way to address the degree of overlap of their pathways is through the MetaCyc pathway ontology. We performed enrichment/depletion analysis in order to determine pathway classes that had sets of matched reactions that were significantly smaller or larger than what might be expected by chance [41]. For determining the pathway classes of MetaCyc that were significantly enriched or depleted for reactions linked to KEGG reactions, we used the Pathway Tools Enrichment Analysis feature. Specifically, we ran the Enrichment Analysis using the exact Fisher method with a maximum p-value of 0.025 and employing the Bonferroni multiple hypothesis testing correction. The results are summarized in Table 11 for MetaCyc pathway classes, and in Table 12 for KEGG pathway classes. Complete results are available in Additional file 2 .

We also asked whether the pathways that are unique to MetaCyc have a taxonomic bias. MetaCyc curators associate an approximate "taxonomic range" for a pathway based on their assessment that most species within the taxonomic range are likely to contain the pathway. The Taxonomic-Range slot lists one or more particular organisms or higher-rank taxa such as phyla or kingdoms. Table 13 scores a MetaCyc pathway as unique if one-third or fewer of the pathway's reactions have links to KEGG

Table 4 Comparison of biochemical reactions in MetaCyc and KEGG

\begin{tabular}{|c|c|c|c|c|c|c|c|}
\hline Category & M(all) & M(base) & M(super) & K(all) & $\mathrm{K}$ (module) & $\mathrm{K}(\mathrm{map})$ & Common \\
\hline All reactions & 10262 & & & 8692 & & & $3895(0.26)$ \\
\hline Pathway reactions & 6348 & 6155 & 6348 & 6174 & 878 & 6173 & $1961(0.19)$ \\
\hline
\end{tabular}


Table 6 A comparison of MetaCyc (M) and KEGG (K) reaction attributes, for those attributes where one hundred or more objects have a value for that attribute

\begin{tabular}{lcll}
\hline $\begin{array}{l}\text { MetaCyc } \\
\text { Attribute (M) }\end{array}$ & Frequency (M) & $\begin{array}{l}\text { KEGG } \\
\text { Attribute (K) }\end{array}$ & Frequency (K) \\
\hline $\begin{array}{l}\text { Physiologically- } \\
\text { Relevant? }\end{array}$ & 10262 & & \\
Creation-Date & 10247 & & \\
& & & \\
Creator & 8090 & & 7632 \\
EC-Number & 7998 & Enzyme & \\
Reaction- & 6660 & & \\
Direction & & \\
Orphan? & 5967 & \\
Credits & 2779 & \\
Rxn-Locations & 282 & \\
Spontaneous? & 238 &
\end{tabular}

Attributes are sorted based on the MetaCyc frequency column. Attribute Physiologically-Relevant? describes whether a reaction occurs in vivo. Reaction-Direction specifies the directionality of the reaction. Orphan? is true when no nucleotide or amino-acid sequence has been determined for any enzyme catalyzing this reaction $[39,40]$. Rxn-Locations specifies the cellular locations in which a reaction occurs (e.g., cytoplasm or mitochondrion). Spontaneous? specifies whether a reaction occurs spontaneously in living organisms and therefore requires no enzyme. KEGG reaction attribute data are derived from the KEGG REACTION dataset, and thus include glycan reactions.

reactions within the same KEGG pathway. Only MetaCyc taxa with 50 or more corresponding MetaCyc pathways are included. An equivalent analysis was not performed using KEGG pathways, as they do not contain taxonomic information in their MAP or MODULE datasets.

\section{Discussion}

\section{Compounds}

Table 1 shows that both DBs contain significant numbers of compounds that are not substrates in any reaction, e.g., 8, 249 of the compounds in KEGG do not directly participate in any reaction; 3,100 MetaCyc compounds do not directly participate in any reaction. MetaCyc includes such compounds for a variety of reasons: some such compounds are activators, inhibitors, and cofactors of MetaCyc enzymes; others are analogs of reaction substrates;

Table 7 Comparison of metabolic pathways, average reactions per pathway, and average compounds per pathway in MetaCyc (M) and KEGG (K)

\begin{tabular}{lcccc}
\hline Category & M(base) & K(module) & M(super) & K(map) \\
\hline Pathway count & 1846 & 179 & 296 & 237 \\
$\begin{array}{l}\text { Reactions per } \\
\text { pathway }\end{array}$ & 4.37 & 6.22 & 14.24 & 28.84 \\
$\begin{array}{l}\text { Compounds per } \\
\text { pathway }\end{array}$ & 11.49 & 15.27 & 25.63 & 37.45 \\
\hline
\end{tabular}

Table 8 Comparison of pathway data content in MetaCyc and KEGG

\begin{tabular}{lcc}
\hline Category & MetaCyc & KEGG \\
\hline Pathway classes & 490 & 107 \\
Pathway instances & 2142 & 416 \\
Pathways with comments & 2122 & 51 \\
Mean comment length & 2240.6 & 83.6 \\
DB links per pathway & 0.34 & 0.88 \\
Reactions per pathway & 5.73 & 19.10
\end{tabular}

KEGG pathway classes were extracted from the MAP and MODULE datasets based on the CLASS attribute. Comment length is measured in number of characters.

others are expected to be present in reactions that will be curated in the future; still others are indirect substrates of MetaCyc reactions because they are instances of MetaCyc compound classes that are substrates of MetaCyc generic reactions. Although users might not expect pathway DBs to contain metabolites that are not participants in pathways or reactions, these metabolites may be useful for identification of compounds from metabolomics datasets.

KEGG contains more duplicate compound entries than does MetaCyc, but overall compound duplicates are relatively low in both DBs.

MetaCyc provides a richer set of compound data fields than KEGG does, including SMILES [43] and InChI [31] strings for most compounds (SMILES is also an ASCII system for encoding chemical structures). In addition, MetaCyc compounds are cross-referenced to the enzymes for which they are activators, inhibitors, and cofactors.

KEGG contains 2.0 times more compounds with comments than does MetaCyc, but the KEGG comments are extremely short, averaging 6.5 characters per comment. MetaCyc comments average 47.7 characters in length. Many KEGG comments are single phrases such as "pesticide".

MetaCyc contains 2.4 names per compound compared to 1.6 for KEGG, which may render MetaCyc more able to recognize chemical names in chemical datasets that use non-standard nomenclature (e.g., metabolomics datasets). On the other hand, KEGG does contain significantly more compounds than MetaCyc.

\section{Reactions}

As noted earlier, many metabolites within the two DBs are not substrates of any reaction; similarly, many reactions within the two DBs are not components of any pathway. This situation occurs for a variety of reasons. Biologically, many metabolic reactions have not been assigned to a metabolic pathway. MetaCyc attempts 


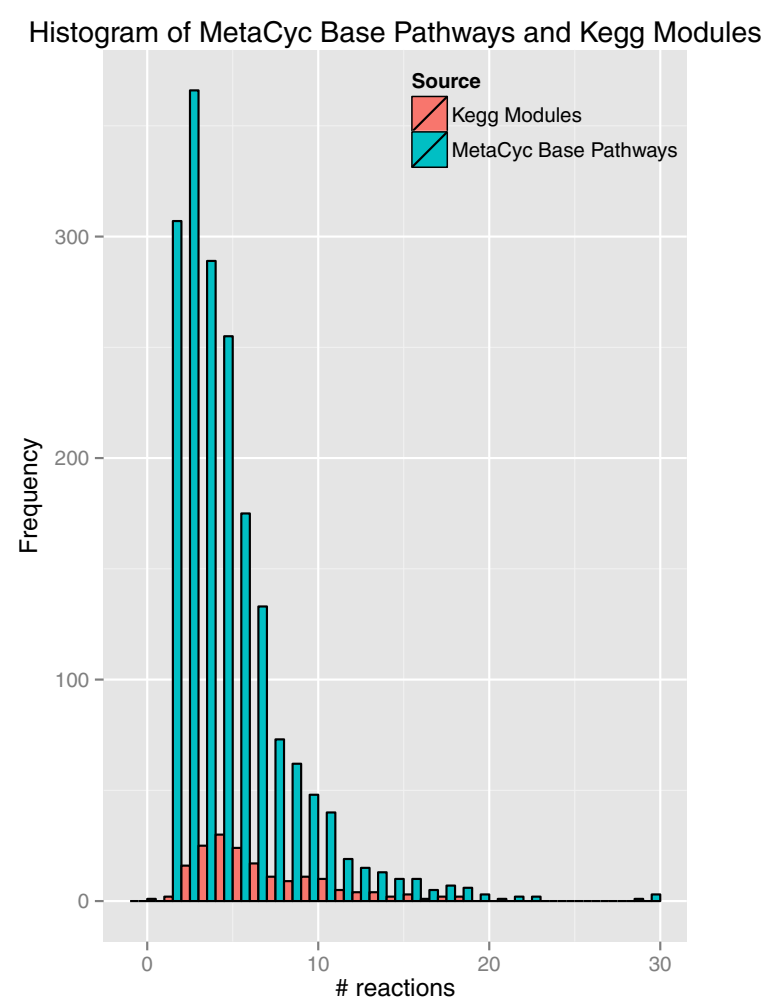

Figure 1 A histogram plot of MetaCyc base pathway and KEGG module size by reaction counts. We excluded one outlier consisting of a MetaCyc base pathway (PWYG-321, "mycolate biosynthesis") with 192 reactions; 17\% of MetaCyc base pathways consist of a single reaction.

to gather a comprehensive compendium of bioreactions for applications such as flux-balance analysis and design of novel metabolic pathways, that do not depend soley on reactions within defined metabolic pathways. In addition, some reactions in MetaCyc and KEGG will probably be assigned to pathways curated in the future.

Overall, MetaCyc contains 1.2 times as many reactions as does KEGG. Applications such as flux-balance analysis require reactions that are fully balanced (including hydrogen) because unbalanced reactions violate conservation of mass and thus the model can generate non-physical flux values. MetaCyc curators routinely encounter unbalanced reactions in the literature, and although many such unbalanced reactions can be corrected by curators, for some unbalanced reactions it is not clear how to correct them.

We can calculate for each DB the number of "high quality reactions" by subtracting from each total the duplicate reactions, and the unbalanced reactions. The results are MetaCyc: 9,451 and KEGG: 6,900, a ratio of 1.37:1.

MetaCyc also provides a richer set of attributes for reactions than does KEGG, such as identification of spontaneous reactions.
The atom mapping of a reaction describes for each reactant non-hydrogen atom its corresponding atom in a product compound. KEGG has provided atom-mapping data through its RPAIR attribute for several years; the Feb 2012 version of KEGG contains atom-mapping data for 8,292 reactions. MetaCyc began providing atom mapping data in version 16.5 in November 2012, which contains atom-mapping data for 8,281 reactions.

Although both DBs employ generic reactions, some details of the treatment of these reactions differ. Generic reactions are reactions in which one or more substrates denote a set of possible compounds, often by using R-groups. For example, the MetaCyc reaction DEOXYCYTIDINE-KINASE-RXN describes the reaction

deoxycytidine + a nucleoside triphosphate $\rightarrow$ dCMP + a nucleoside diphosphate

KEGG contains the same reaction (R02321) with the same equation. However, KEGG represents the compound classes differently. In MetaCyc "a nucleoside triphosphate" is described by a class frame (Nucleoside-Triphosphates). The MetaCyc ontology links that class frame to several subclasses, and ultimately to eleven specific compounds that are instances 


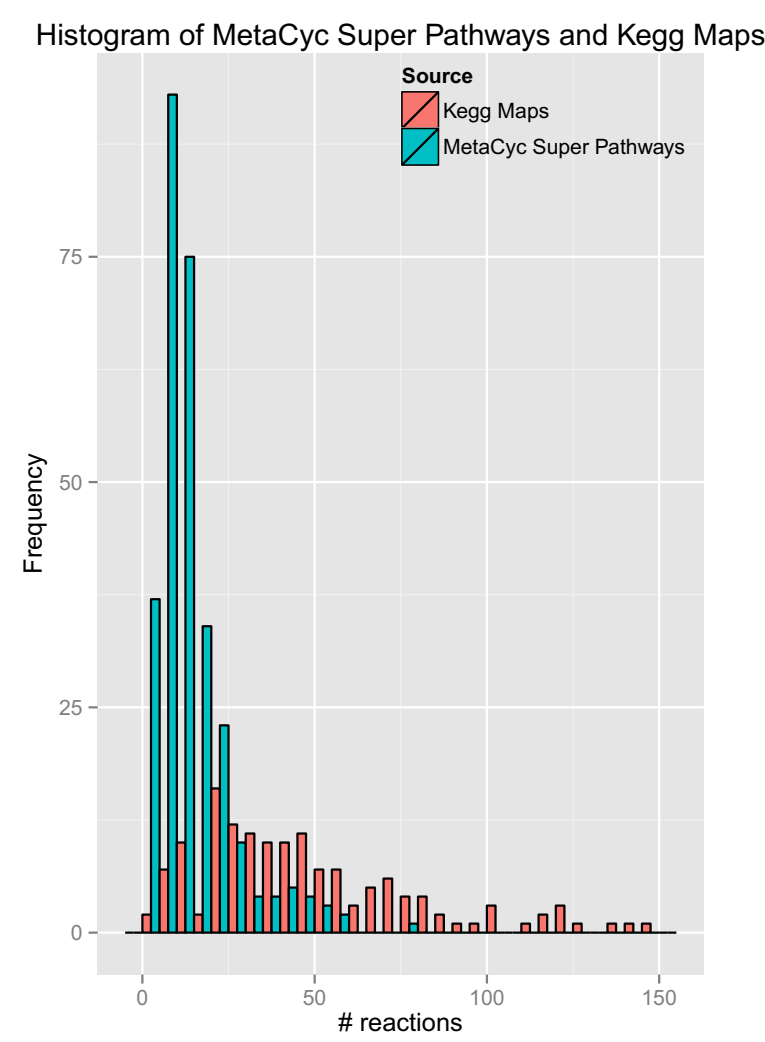

Figure 2 A histogram plot of MetaCyc super pathway and KEGG map size by reaction counts. We excluded one outlier consisting of a MetaCyc super pathway (PWY-6113, "mycolate biosynthesis") with 233 reactions.

of that class, such as ATP. This representation allows software within Pathway Tools to generate instantiations of generic reactions - namely, to generate all possible instance reactions (reactions all of whose substrates are instance compounds, not classes) that are specializations of the generic reaction. MetaCyc contains 2,884 generic reactions, from which many additional reactions can be generated through instantiation. In contrast, although KEGG contains an object representing the generic compound (C00201), that generic compound is not found in the KEGG BRITE ontology, nor does KEGG contain links from the generic compound to instances of that compound. Thus, so far as we know, the KEGG representations do not facilitate programmatic instantiation of generic reactions.

\section{Pathways}

Based on Table 7, MetaCyc contains 10.3 times as many base pathways as KEGG contains modules. MetaCyc contains 1.2 times as many superpathways as KEGG contains maps. Because pathway size measured in reactions varies so strongly between the two DBs, comparing the DBs purely based on pathway counts can be misleading - the average MetaCyc base pathway contains
4.37 reactions, whereas the average KEGG map contains 28.84 reactions. Furthermore, $17 \%$ of MetaCyc pathways consist of a single reaction step - namely, in those cases where the MetaCyc curation rules on defining pathway boundaries $[44,45]$ result in single-reaction pathways.

A more meaningful way to compare the pathway complements of the two DBs is to compare the size of the metabolite and reaction spaces covered by these pathways. Table 1 shows that MetaCyc pathways refer to 5,523 distinct metabolites, or 1.16 times as many as KEGG. A small difference exists between the substrates covered by MetaCyc base pathways versus MetaCyc super pathways, most likely because MetaCyc super pathways are ultimately defined in terms of base pathways, plus some additional reactions not present in the base pathways. In contrast, there is a large difference between the substrates covered by KEGG maps versus modules - modules cover a very small set of substrates compared to maps and compared to MetaCyc pathways.

We posit that KEGG has such a small number of modules because modules were introduced to KEGG in the last few years, and their coverage is still limited. For 
Table 9 A comparison of MetaCyc (M) and KEGG (K) pathway attributes, for those attributes where one hundred or more objects have a value for that attribute

\begin{tabular}{lclc}
\hline $\begin{array}{l}\text { MetaCyc } \\
\text { Attribute (M) }\end{array}$ & Frequency (M) & $\begin{array}{l}\text { KEGG } \\
\text { Attribute (K) }\end{array}$ & Frequency (K) \\
\hline Species & 2141 & & \\
Pathway-Links & 1412 & Rel_Pathway & 345 \\
Creation-Date & 2139 & & \\
Taxonomic-Range & 2135 & & \\
Creator & 2092 & & 154 \\
Predecessors & 2089 & ECrel & \\
Credits & 1944 & & 220 \\
Key-Reactions & 373 & & \\
& & Disease & \\
Hypothetical- & 105 & & \\
Reactions & &
\end{tabular}

Attributes are sorted based on frequency. KEGG pathway attribute data are pooled from all objects in the KEGG MODULE and MAP datasets (which include data from global pathways and pathway classes with no metabolic reaction data). Attribute Species specifies the organisms in which the pathway has been studied experimentally. Pathway-Links lists important substrates that connect to other metabolic pathways, whereas KEGG attribute Rel_Pathway links pathways to one another without specifying the compound in common.

Taxonomic-Range describes the taxonomic groups in which the pathway is likely to be found; this information increases the accuracy of pathway prediction. Predecessors specifies for each reaction the reaction(s) that precede it in the pathway, and thus defines the connectivity structure of each pathway. KEGG encodes equivalent data in the "ECrel" relationship, obtained from the get_element_relations_by_pathway API function. Key-Reactions increases the accuracy of pathway prediction by specifying reactions whose presence is highly indicative of the pathway, and distinguish the pathway from other, similar pathways. Hypothetical-Reactions identifies pathway reactions that are speculative and have not been firmly established experimentally. The Disease attribute consists of links to the KEGG DISEASE dataset when disease-related genes encode enzymes for one or more reaction steps in the pathway.

example, KEGG contains one module for proline biosynthesis; MetaCyc contains four such pathways. KEGG lacks modules for biosynthesis of the amino acids valine, glycine, aspartate, alanine, glutamine, and glutamate (most but not all are one reaction pathways). That MetaCyc contains 10.3 times as many base pathways as KEGG contains modules means that studies such as [46] that analyze the pathway content of metagenomic samples may be incomplete because they may miss pathways using the limited repertoire of KEGG modules that could be found using MetaCyc base pathways (note that [46] also included KEGG maps in their analysis).

MetaCyc pathways refer to 6,348 reactions, or 1.03 times as many reactions as referred to in KEGG pathways. Thus, the reaction spaces covered by the two DBs are very similar in size.

MetaCyc provides a more extensive array of pathway attributes than does KEGG. Some of these attributes can be used to increase the accuracy of pathway prediction, e.g., Taxonomic-Range and Key-Reactions. Lacking those attributes, pathway predictions performed using KEGG pathways are likely to be less accurate than for MetaCyc pathways.

In the years before KEGG introduced its modules, KEGG and MetaCyc employed very different conceptualizations of pathways. As discussed in detail in [45], KEGG maps are larger than MetaCyc base pathways because KEGG maps are mosaics that integrate reactions from multiple organisms and multiple biological pathways. For example, KEGG map00270 ("cysteine and methionine metabolism") integrates reactions from pathways involving the biosynthesis of both L-cysteine and L-methionine, and their conversion to compounds such as L-cystathionine and L-homocysteine, from all domains of life. In contrast, MetaCyc creates separate base pathways - called pathway variants - for each distinct pathway of L-methionine biosynthesis (eight pathways) and L-cysteine biosynthesis (four pathways) that has been experimentally elucidated in a given organism (pathways are considered distinct if they contain different sets of reactions). MetaCyc pathway boundaries are defined [45] based on evolutionary conservation, on the metabolism literature, on regulation, and on stable high-connectivity metabolites. We estimate that KEGG modules are created according to principles similar to those of MetaCyc base pathways.

These differences in pathway conceptualization have different implications, depending on the intended uses of pathway data. (1) MetaCyc pathways (and probably KEGG modules) more accurately portray the exact biological pathways that occur in a specific organism, because for a KEGG map, its mosaic nature means that the user cannot tell which subset of its reactions was experimentally elucidated in a particular organism. (2) KEGG maps (and MetaCyc superpathways) are more effective at portraying the set of possible reactions that can impinge on a given metabolite in a wide range of organisms. (3) KEGG maps are not effective for statistical correlation studies because they encompass so much metabolic ground. For example, if we compare two metagenomic datasets and find that map00270 ("cysteine and methionine metabolism") is present in one but not the other, is it the biosynthesis

Table 10 Degree to which pathways in MetaCyc (M) and KEGG (K) have their reactions linked to the other DB

\begin{tabular}{lcccc}
\hline $\begin{array}{l}\text { Reaction } \\
\text { Coverage }\end{array}$ & M(base) & K(module) & M(super) & K(map) \\
\hline $\begin{array}{l}\text { All reactions } \\
\text { linked }\end{array}$ & 549 & 73 & 0 & 3 \\
$\begin{array}{l}\text { Some reactions } \\
\text { linked }\end{array}$ & 731 & 80 & 73 & 128 \\
$\begin{array}{l}\text { No reactions } \\
\text { linked }\end{array}$ & 566 & 26 & 223 & 106 \\
\hline
\end{tabular}

For example, for three KEGG maps, all reactions in the pathway are present in MetaCyc. 
Table 11 MetaCyc pathway classes that are significantly enriched or depleted for reactions with links to KEGG

\begin{tabular}{|c|c|c|c|c|}
\hline Status & Pathway class & Class size & Links & p-value \\
\hline Enriched & Amino Acids Biosynthesis & 112 & $\frac{186}{260}$ & $1.4 \times 10^{-20}$ \\
\hline Enriched & Individual Amino Acids Biosynthesis & 99 & $\frac{174}{244}$ & $4.0 \times 10^{-19}$ \\
\hline Enriched & Amino Acids Degradation & 118 & $\frac{222}{326}$ & $2.0 \times 10^{-17}$ \\
\hline Enriched & Purine Nucleotide Biosynthesis & 19 & $\frac{46}{56}$ & $3.2 \times 10^{-10}$ \\
\hline Enriched & Generation Of Precursor Metabolites And Energy & 162 & $\frac{170}{304}$ & $2.6 \times 10^{-9}$ \\
\hline Enriched & C1 Compounds Utilization And Assimilation & 28 & $\frac{75}{102}$ & $9.3 \times 10^{-9}$ \\
\hline Enriched & Autotrophic $\mathrm{CO}_{2}$ Fixation & 7 & $\frac{47}{57}$ & $1.3 \times 10^{-7}$ \\
\hline Enriched & $\mathrm{CO}_{2}$ Fixation & 9 & $\frac{48}{59}$ & $2.8 \times 10^{-7}$ \\
\hline Enriched & Vitamins Biosynthesis & 68 & $\frac{128}{223}$ & $1.4 \times 10^{-6}$ \\
\hline Enriched & Sugar Derivatives Degradation & 42 & $\frac{109}{162}$ & $3.8 \times 10^{-6}$ \\
\hline Enriched & Sugar Alcohols Degradation & 12 & $\frac{54}{68}$ & $6.0 \times 10^{-6}$ \\
\hline Enriched & Amines And Polyamines Biosynthesis & 37 & $\frac{57}{74}$ & $6.9 \times 10^{-6}$ \\
\hline Enriched & Carboxylates Degradation & 44 & $\frac{82}{132}$ & $1.0 \times 10^{-5}$ \\
\hline Enriched & Sugars Degradation & 51 & $\frac{108}{162}$ & $1.5 \times 10^{-5}$ \\
\hline Enriched & NAD Biosynthesis & 8 & $\frac{23}{23}$ & $1.6 \times 10^{-5}$ \\
\hline Enriched & Fermentation & 46 & $\frac{75}{106}$ & $3.6 \times 10^{-5}$ \\
\hline Enriched & Nucleosides And Nucleotides Biosynthesis & 35 & $\frac{69}{128}$ & $6.8 \times 10^{-5}$ \\
\hline Enriched & Nucleosides And Nucleotides Degradation & 29 & $\frac{64}{90}$ & $1.4 \times 10^{-4}$ \\
\hline Enriched & Purine Nucleotide Salvage & 13 & $\frac{26}{28}$ & $1.7 \times 10^{-4}$ \\
\hline Enriched & Arginine Degradation & 15 & $\frac{35}{42}$ & $4.6 \times 10^{-4}$ \\
\hline Enriched & Purine Nucleotide De Novo Biosynthesis & 6 & $\frac{22}{30}$ & $4.9 \times 10^{-4}$ \\
\hline Enriched & Mandelates Degradation & 2 & $\frac{18}{18}$ & $9.9 \times 10^{-4}$ \\
\hline Enriched & Gluconeogenesis & 2 & $\frac{23}{28}$ & $1.1 \times 10^{-3}$ \\
\hline Enriched & Glycolysis & 6 & $\frac{27}{30}$ & $2.0 \times 10^{-3}$ \\
\hline Enriched & NAD Metabolism & 11 & $\frac{28}{33}$ & $2.1 \times 10^{-3}$ \\
\hline Enriched & Geranylgeranyldiphosphate Biosynthesis & 3 & $\frac{18}{18}$ & $2.3 \times 10^{-3}$ \\
\hline Enriched & Catechol Degradation & 7 & $\frac{17}{17}$ & $2.3 \times 10^{-3}$ \\
\hline Enriched & Methionine Biosynthesis & 13 & $\frac{29}{34}$ & $4.0 \times 10^{-3}$ \\
\hline Enriched & Photosynthesis & 5 & $\frac{24}{30}$ & $4.0 \times 10^{-3}$ \\
\hline Enriched & Pyrimidine Nucleotide Biosynthesis & 8 & $\frac{36}{53}$ & $5.7 \times 10^{-3}$ \\
\hline Enriched & Toluenes Degradation & 13 & $\frac{35}{46}$ & $7.2 \times 10^{-3}$ \\
\hline Enriched & Glutamate Degradation & 10 & $\frac{28}{35}$ & $1.5 \times 10^{-2}$ \\
\hline Enriched & Formaldehyde Assimilation & 3 & $\frac{24}{28}$ & $1.6 \times 10^{-2}$ \\
\hline Enriched & Alcohols Degradation & 17 & $\frac{24}{30}$ & $1.6 \times 10^{-2}$ \\
\hline Enriched & Urate Degradation & 2 & $\frac{17}{18}$ & $2.4 \times 10^{-2}$ \\
\hline Enriched & Cobalamin Biosynthesis & 9 & $\frac{35}{46}$ & $2.5 \times 10^{-2}$ \\
\hline Depleted & Secondary Metabolites Biosynthesis & 460 & $\frac{579}{1896}$ & $3.8 \times 10^{-35}$ \\
\hline Depleted & Glucosinolates Biosynthesis & 9 & $\frac{4}{104}$ & $2.0 \times 10^{-17}$ \\
\hline Depleted & Biosynthesis & 1182 & $\frac{1459}{4215}$ & $2.3 \times 10^{-16}$ \\
\hline Depleted & Nitrogen Containing Glucosides Biosynthesis & 13 & $\frac{11}{125}$ & $8.0 \times 10^{-15}$ \\
\hline Depleted & Hormones Degradation & 24 & $\frac{12}{124}$ & $2.7 \times 10^{-13}$ \\
\hline Depleted & Polymeric Compounds Degradation & 35 & $\frac{17}{136}$ & $3.5 \times 10^{-12}$ \\
\hline Depleted & Polysaccharides Degradation & 33 & $\frac{17}{127}$ & $2.0 \times 10^{-10}$ \\
\hline Depleted & Steroids Degradation & 8 & $\frac{2}{46}$ & $4.2 \times 10^{-6}$ \\
\hline
\end{tabular}


Table 11 MetaCyc pathway classes that are significantly enriched or depleted for reactions with links to KEGG (Continued)

\begin{tabular}{lllll}
\hline Depleted & Polyketides Biosynthesis & 13 & $\frac{6}{62}$ & $1.5 \times 10^{-5}$ \\
Depleted & Glucosinolates Degradation & 4 & $\frac{0}{30}$ & $7.0 \times 10^{-5}$ \\
Depleted & Cholesterol Degradation & 4 & $\frac{1}{33}$ & $3.3 \times 10^{-4}$ \\
Depleted & Fatty Acid Biosynthesis & 49 & $\frac{20}{354}$ & $3.4 \times 10^{-4}$ \\
Depleted & Nitrogen Containing Secondary Compounds Degradation & 18 & $\frac{13}{77}$ & $1.0 \times 10^{-3}$ \\
Depleted & Terpenoids Biosynthesis & 127 & $\frac{176}{530}$ \\
Depleted & Plant Hormones Degradation & 15 & $\frac{7}{55}$ & $1.2 \times 10^{-3}$ \\
Depleted & Sesquiterpenoids Biosynthesis & 32 & $\frac{25}{114}$ & $1.6 \times 10^{-3}$ \\
Depleted & Chlorotoluene Degradation & 5 & $\frac{0}{24}$ & $1.6 \times 10^{-3}$ \\
Depleted & Auxins Degradation & 8 & $\frac{0}{23}$ & $2.3 \times 10^{-3}$ \\
Depleted & Apocarotenoids Biosynthesis & 4 & $5.1 \times 10^{-3}$ \\
Depleted & Lignans Biosynthesis & 5 & $\frac{0}{20}$ & $\frac{0}{20}$ \\
\hline
\end{tabular}

Class size is the number of pathway instances for the given pathway class. The 'Links' column is the number of reactions among the pathways of the pathway class that have links to KEGG reactions, over the total number of reactions for the pathway class. The Bonferroni-corrected p-value from the hypergeometric test indicates the probability that the observed proportion of reactions with links within the pathway occurred by chance. Pathways with a p-value at or below a cut-off of $\alpha=0.025$ are shown. The full list may be found in the Additional file 2 .

Table 12 KEGG pathway classes that are significantly enriched or depleted for reactions with links to MetaCyc

\begin{tabular}{|c|c|c|c|c|}
\hline Status & Pathway class & Class size & Links & p-value \\
\hline Enriched & Nucleotide And Amino Acid Metabolism & 72 & $\frac{346}{419}$ & $2.6 \times 10^{-56}$ \\
\hline Enriched & Carbohydrate Metabolism & 15 & $\frac{496}{766}$ & $4.1 \times 10^{-29}$ \\
\hline Enriched & Amino Acid Metabolism & 13 & $\frac{478}{784}$ & $3.0 \times 10^{-20}$ \\
\hline Enriched & Energy Metabolism & 8 & $\frac{167}{246}$ & $2.9 \times 10^{-14}$ \\
\hline Enriched & Cofactor And Vitamin Biosynthesis & 19 & $\frac{104}{133}$ & $1.8 \times 10^{-11}$ \\
\hline Enriched & Energy Metabolism & 24 & $\frac{89}{114}$ & $1.3 \times 10^{-10}$ \\
\hline Enriched & Carbon Fixation & 13 & $\frac{56}{66}$ & $7.6 \times 10^{-8}$ \\
\hline Enriched & Aromatic Amino Acid Metabolism & 11 & $\frac{45}{55}$ & $2.2 \times 10^{-5}$ \\
\hline Enriched & Alkaloid And Other Secondary Metabolite Biosynthesis & 4 & $\frac{28}{30}$ & $2.9 \times 10^{-5}$ \\
\hline Enriched & Other Carbohydrate Metabolism & 6 & $\frac{37}{44}$ & $2.9 \times 10^{-5}$ \\
\hline Enriched & Nucleotide Metabolism & 2 & $\frac{159}{261}$ & $7.6 \times 10^{-5}$ \\
\hline Enriched & Cysteine And Methionine Metabolism & 6 & $\frac{27}{30}$ & $3.4 \times 10^{-4}$ \\
\hline Enriched & Central Carbohydrate Metabolism & 13 & $\frac{42}{52}$ & $4.4 \times 10^{-4}$ \\
\hline Enriched & Reaction Motif & 3 & $\frac{15}{15}$ & $7.1 \times 10^{-3}$ \\
\hline Enriched & Arginine And Proline Metabolism & 3 & $\frac{15}{15}$ & $7.1 \times 10^{-3}$ \\
\hline Enriched & Histidine Metabolism & 2 & $\frac{14}{14}$ & $1.6 \times 10^{-2}$ \\
\hline Enriched & Purine Metabolism & 3 & $\frac{23}{28}$ & $2.1 \times 10^{-2}$ \\
\hline Depleted & Xenobiotics Biodegradation And Metabolism & 20 & $\frac{258}{1013}$ & $1.0 \times 10^{-37}$ \\
\hline Depleted & Glycan Biosynthesis And Metabolism & 15 & $\frac{36}{254}$ & $1.9 \times 10^{-21}$ \\
\hline Depleted & Metabolism Of Terpenoids And Polyketides & 20 & $\frac{265}{848}$ & $4.4 \times 10^{-13}$ \\
\hline Depleted & Glycan Metabolism & 10 & $\frac{0}{51}$ & $6.8 \times 10^{-11}$ \\
\hline Depleted & Glycosaminoglycan Metabolism & 7 & $\frac{0}{30}$ & $2.0 \times 10^{-5}$ \\
\hline Depleted & Lipid Metabolism & 17 & $\frac{245}{713}$ & $1.4 \times 10^{-3}$ \\
\hline
\end{tabular}

Class size is the number of pathway instances for the given pathway class. The 'Links' column is the number of reactions among the pathways of the pathway class that have links to MetaCyc reactions, over the total number of reactions for the pathway class. The Bonferroni-corrected p-value from the hypergeometric test indicates the probability that the observed proportion of reactions with links within the pathway occurred by chance. Pathways with a p-value at or below a cut-off of $\alpha=0.025$ are shown. The full list may be found in the Additional file 2 . 
Table 13 Taxonomic analysis of MetaCyc base pathways that are not represented in KEGG pathways

\begin{tabular}{|c|c|c|c|c|}
\hline ID & Taxon & Pathways & Unique Pathways & $\%$ Unique \\
\hline 131567 & Cellular Organisms & 1840 & 878 & 47.7 \\
\hline 2759 & Eukaryota & 1094 & 512 & 46.8 \\
\hline 33090 & Viridiplantae (green plants) & 650 & 348 & 53.5 \\
\hline 35493 & Streptophyta & 374 & 222 & 59.4 \\
\hline 3193 & Embryophyta (plants) & 373 & 221 & 59.2 \\
\hline 58023 & Tracheophyta (vascular plants) & 298 & 181 & 60.7 \\
\hline 78536 & Euphyllophyta & 289 & 175 & 60.6 \\
\hline 58024 & Spermatophyta (seed plants) & 285 & 174 & 61.1 \\
\hline 3398 & Magnoliophyta (flowering plants) & 262 & 168 & 64.1 \\
\hline 91827 & Core Eudicotyledons & 162 & 108 & 66.7 \\
\hline 71275 & Rosids & 100 & 71 & 71.0 \\
\hline 33154 & Opisthokonta & 351 & 131 & 37.3 \\
\hline 33208 & Metazoa (multicellular animals) & 129 & 47 & 36.4 \\
\hline 7711 & Chordata & 54 & 20 & 37.0 \\
\hline 7742 & Vertebrata & 52 & 20 & 38.5 \\
\hline 4751 & Fungi & 219 & 78 & 35.6 \\
\hline 2 & Bacteria & 1040 & 426 & 41.0 \\
\hline 201174 & Actinobacteria & 72 & 37 & 51.4 \\
\hline 1224 & Proteobacteria (purple photosynthetic bacteria) & 169 & 64 & 37.9 \\
\hline 2157 & Archaea & 209 & 82 & 39.2 \\
\hline
\end{tabular}

The ID column is the NCBI Taxonomy DB [42] identifier. The pathways column is the number of MetaCyc pathways that occur in that taxon based on its Taxonomic-Range slot. The unique pathways column is the number of MetaCyc base pathways for that taxon that are unique relative to KEGG pathways. The percent unique column is the fraction of MetaCyc base pathways for that taxon that are unique relative to KEGG pathways, with rows with a fraction greater than $50 \%$ shown in bold. The rows of the table are sorted with respect to the NCBI Taxonomy. Relative taxonomic rank is indicated by indentation. Taxa of the same rank are ordered by decreasing percent unique pathways. The taxon of "Cellular Organisms" is included to provide a baseline from which to compare other taxa.

of cysteine that is over represented, or that of methionine? Or is it the biosynthesis of other compounds in this map (such as L-cystathionine and L-homocysteine) that are over represented? Abubucker et al. make a similar point [46] about KEGG maps. (4) We argue that for pathway reconstruction in sequenced genomes, MetaCyc pathways are more effective because their smaller size produces more focused predictions. For example, KEGG shows its map00680 ("methane metabolism") as present in E. coli K-12 MG1655 with 23 reactions (excluding transporters) colored as occurring in this organism. Yet, E. coli K-12 MG1655 does not produce methane. A counter-example of KEGG pathway prediction comes from the photosynthesis map (map00195), for which only annotations based on photosynthetic organisms can be selected on the KEGG website. Thus, it is unclear what rules KEGG uses to call a given map as present or absent in a given organism; the rules used by Pathway Tools are published [47].

When a map is called as present by KEGG, does it predict all reactions in the map as present in that organism? For example, for the methane metabolism pathway in E. coli, are the additional 55 uncolored reactions inferred as present in textitE. coli? Since KEGG pathways are known to be multi-organism mosaics, such an inference will surely contain many false-positive reactions. In contrast, when a MetaCyc pathway is predicted as present, the assertion is that all of its reactions are probably present, permitting a more focused and accurate prediction of the reactome of an organism. This more accurate prediction of the reactome has implications for metabolic modeling using flux-balance analysis, where missing reactions usually yield non-solvable models, whereas extra reactions can yield models that make erroneous predictions. KEGG may resolve these issues once its collection of modules is more extensive, but currently its modules cover too little of metabolism to have broad utility.

Figure 1 and Figure 2 reveal that while MetaCyc base pathways have a distribution range comparable to that of KEGG modules, there is a significant difference in mean and variance for MetaCyc super pathways and KEGG maps. Many pathway analyses, such as enrichment/depletion, may exhibit bias when the sets of pathways have a large range of sizes. By virtue of having a smaller range of sizes, MetaCyc super pathways provide a more consistent basis for performing pathway analyses. 
We analyzed the degree of overlap on a pathway-class basis in Tables 11 and 12, revealing the pathway classes that are enriched for reaction links (i.e., there is a significant amount of overlap between the two databases), and the pathway classes that are depleted for reaction links (i.e., the pathway class is relatively unique to its database). The KEGG pathway class depletion in Table 12 shows that the metabolism of MetaCyc is under-represented for counterparts of the KEGG maps for xenobiotics, glycans, and polyketides. For glycans and polyketides, we expect that this is because MetaCyc does not currently have the ability to represent abstracted versions of glycan chemical structures, nor abstracted versions of polyketide pathways, found in KEGG map drawings.

Table 13 shows that MetaCyc contains large numbers of unique pathways, which are primarily found in plant taxa, but are also found in vertebrates, chordata, and metazoa; in fungi; in archaea; and in proteobacteria.

\section{Miscellaneous}

MetaCyc contains extensive data on metabolic enzymes. Version 16.0 of MetaCyc contains 7,893 metabolic enzymes. MetaCyc describes enzyme subunit composition, substrate specificity, activators, inhibitors, and cofactor requirements. KEGG does not describe the protein properties of metabolic enzymes, and therefore lacks this type of data; KEGG does associate cofactors with reactions.

MetaCyc and KEGG also differ in their licensing terms. MetaCyc data are freely available to all users via data file download in multiple formats, and may be openly redistributed. KEGG dataset FTP downloads are available for a fee to all users, and may not be openly redistributed. KEGG provides a web service API for requesting entries individually, as does MetaCyc.

\section{Conclusions}

We have compared the contents of the KEGG and MetaCyc pathway DBs. Because pathway DBs contain multiple types of data, our comparison is necessarily multidimensional. KEGG contains significantly more compounds than does MetaCyc, whereas MetaCyc contains significantly more reactions and pathways than does KEGG. However, the number of reactions occurring in pathways, 6,348 for MetaCyc and 6,174 for KEGG, are quite similar. Only 1,961 of those reactions have been identified as reactions shared by the two DBs. We expect that as many as 1,636 additional shared reactions will be found, which would leave a substantial number of reactions that are unique to each DB. We estimated the set of pathways that are present in one DB but not in the other DB, and found that MetaCyc pathways not found in KEGG are predominately from plants, fungi, metazoa, and actinobacteria; KEGG pathways not found in MetaCyc are for xenobiotic degradation, glycan metabolism, and metabolism of terpenoids and polyketides. MetaCyc contains more reactions that are fully balanced, which facilitates metabolic modeling such as using flux-balance analysis. MetaCyc includes generic reactions that may be instantiated computationally. Both databases contain atom-mapping data.

\section{Additional files}

Additional file 1: The software used in building the KeggCyc DB is available for use with Pathway Tools as a Common Lisp source code file.

Additional file 2: The full results of the enrichment/depletion analysis may be found in the additional file in a spreadsheet file.

\section{Competing interests}

The authors benefit financially from commercial licensing of the Pathway Tools software.

\section{Authors' contributions}

TA authored substantial text, and performed most of the comparisons and analyses. MT authored the KeggCyc loader software. AK curated and checked many correspondences between KEGG and MetaCyc. RC commented on the manuscript and provided valuable discussions on many issues. PDK supervised the research and authored substantial text. All authors read and approved the final manuscript.

\section{Acknowledgements}

We thank Joseph M. Dale for helpful discussions on the Jaccard coefficient, the Tanimoto coefficient, and cosine similarity measures.

This work was supported by award number R01GM080746 from the National Institute of General Medical Sciences of the National Institutes of Health; and by grant number DE-SC0004878 from the the Office of Science (BER), U.S. Department of Energy. The content of this article is solely the responsibility of the authors and does not necessarily represent the official views of the National Institutes of Health, or of the Department of Energy.

Received: 6 July 2012 Accepted: 4 March 2013

Published: 27 March 2013

\section{References}

1. Caspi R, Altman T, Dale JM, Dreher K, Fulcher CA, Gilham F, Kaipa P, Karthikeyan AS, Kothari A, Krummenacker M, Latendresse M, Mueller LA, Paley S, Popescu L, Pujar A, Shearer AG, Zhang P, Karp PD: The MetaCyc database of metabolic pathways and enzymes and the BioCyc collection of pathway/genome databases. Nuc Acids Res 2010, 38:D473-D479 doi:10.1093/nar/gkp875. advanced access.

2. Caspi R, Altman T, Dreher K, Fulcher CA, Subhraveti $P$, Keseler I, Kothari A, Krummenacker M, Latendresse M, Mueller LA, Ong Q, Paley S, Pujar A, Shearer AG, Travers M, Weerasinghe D, Zhang P, Karp PD: The MetaCyc database of metabolic pathways and enzymes and the BioCyc collection of pathway/genome databases. Nuc Acids Res 2012 , 40:D742-D753.

3. Caspi R, Foerster H, Fulcher CA, Hopkinson R, Ingraham J, Kaipa P, Krummenacker M, Paley S, Pick J, Rhee SY, Tissier C, Zhang P, Karp PD: MetaCyc: A multiorganism database of metabolic pathways and enzymes. Nuc Acids Res 2006, 34:D511-D516.

4. Caspi R, Foerster H, Fulcher CA, Kaipa P, Krummenacker M, Latendresse M, Paley S, Rhee SY, Shearer A, Tissier C, Walk TC, Zhang P, Karp PD: The MetaCyc database of metabolic pathways and enzymes and the BioCyc collection of pathway/genome databases. Nuc Acids Res 2008, 36:D623-D631.

5. Karp PD, Caspi R: A survey of metabolic databases emphasizing the MetaCyc family. Arch of Toxicol 2011, 85:1015-1033.

6. Karp PD, Riley M, Paley S, Pellegrini-Toole A: The MetaCyc database. Nuc Acids Res 2002, 30(1):59-61. 
7. Karp PD, Riley M, Saier M, Paulsen IT, Paley S, Pellegrini-Toole A: The EcoCyc and MetaCyc databases. Nuc Acids Res 2000, 28(1):56-59.

8. Krieger CJ, Zhang P, Mueller LA, Wang A, Paley S, Arnaud M, Pick J, Rhee SY, Karp PD: MetaCyc: A multiorganism database of metabolic pathways and enzymes. Nuc Acids Res 2004, 32:D438-D442.

9. Kanehisa M, Goto S: KEGG: Kyoto Encyclopedia of genes and genomes. Nuc Acids Res 2000, 28:27-30.

10. Kanehisa M, Goto S, Furumichi M, Tanabe M, Hirakawa M: KEGG for representation and analysis of molecular networks involving diseases and drugs. Nuc Acids Res 2010, 38(Database issue):D355-D360.

11. Kanehisa M, Goto S, Hattori M, Aoki-Kinoshita KF, Itoh M, Kawashima S, Katayama T, Araki M, Hirakawa M: From genomics to chemical genomics: New developments in KEGG. Nuc Acids Res 2006 34:D354-D357.

12. Kanehisa M, Goto S, Kawashima S, Okuno Y, Hattori M: The KEGG resource for deciphering the genome. NuCAcids Res 2004, 32:D277-D280

13. Kanehisa M, Goto S, Kawashima S, Nakaya A: The KEGG databases at GenomeNet. Nuc Acids Res 2002, 30:42-46.

14. Ogata H, Goto S, Sato K, Fujibuchi W, Bono H, Kanehisa M: KEGG: Kyoto encyclopedia of genes and genomes. Nuc Acids Res 1999, 27(1): 29-34.

15. Okuda S, Yamada T, Hamajima M, Itoh M, Katayama T, Bork P, Goto S, Kanehisa M: KEGG atlas mapping for global analysis of metabolic pathways. Nuc Acids Res 2008, 36:W423-W326.

16. Pathway/Genome Database Websites. [http://BioCyc.org/otherpgdbs. shtml]

17. Dale JM, Popescu L, Karp PD: Machine learning methods for metabolic pathway prediction. BMC Bioinformatics 2010, 11:15

18. Bernard T, Bridge A, Morgat A, Moretti S, Xenarios I, Pagni M: Reconciliation of metabolites and biochemical reactions for metabolic networks. Brief Bioinform 2012. (ENG) http://bib. oxfordjournals.org/content/early/2012/12/03/bib.bbs058.long.

19. Henry CS, DeJongh M, Best AA, Frybarger PM, Linsay B, Stevens RL: High-throughput generation, optimization and analysis of genome-scale metabolic models. Nat Biotechnol 2010, 28(9):977-982.

20. Soh D, Dong D, Guo Y, Wong L: Consistency, comprehensiveness, and compatibility of pathway databases. BMC Bioinformatics 2010, 11:449.

21. Stobbe MD, Houten SM, Jansen GA, van Kampen AH, Moerland PD: Critical assessment of human metabolic pathway databases: $A$ stepping stone for future integration. BMC Syst Biol 2011, 5:165.

22. Chindelevitch L, Stanley S, Hung D, Regev A, Berger B: Metamerge: scaling up genome-scale metabolic reconstructions with application to mycobacterium tuberculosis. Genome Biol 2012, 13(1):r6.

23. Kumar A, Suthers PF, Maranas CD: MetRxn: A knowledgebase of metabolites and reactions spanning metabolic models and databases. BMC Bioinformatics 2012, 13(1):6.

24. Lang $M$, Stelzer $M$, Schomburg D: BKM-react, an integrated biochemical reaction database. BMC Biochem 2011, 12:42.

25. Rajasimha HK: PathMeld: A methodology for the unification of metabolic pathway databases. 2004. Master's thesis. Virginia Polytechnic Institute and State University.

26. Kawashima S, Katayama T, Sato Y, Kanehisa M: KEGG API: A Web Service Using SOAP/WSDL to Access the KEGG System. Genome Informatics 2003, 14:673-674

27. Elhai J, Taton A, Massar JP, Myers JK, Travers M, Casey J, Slupesky M, Shrager J: BioBIKE: a Web-based, programmable, integrated biological knowledge base. Nucleic Acids Res 2009, 37(Web Server issue):28-32.

28. Karp PD, Paley SM, Krummenacker M, Latendresse M, Dale JM, Lee T, Kaipa P, Gilham F, Spaulding A, Popescu L, Altman T, Paulsen I, Keseler IM, Caspi R: Pathway Tools version 13.0: Integrated software for pathway/genome informatics and systems biology. Brief Bioinform 2010, 11:40-79 doi:10.1093/bib/bbp043.

29. Bolton E, Wang Y, Thiessen PA, Bryant SH: PubChem: Integrated Platform of Small Molecules and Biological Activities, vol.4. Washington: American Chemical Society; 2008.

30. Tan P, Steinbach M, Kumar V: Introduction to Data Mining. Boston: Pearson Addison Wesley; 2006

31. Stein SE, Heller S R, Tchekhovskoi D: An open standard for chemical structure representation: The IUPAC chemical identifier. In Proc. 2003 International Chemical Information Conference (Nimes): 2003:131-143.
32. Alcantara R, Axelsen KB, Morgat A, Belda E, Coudert E, Bridge A, Cao H, de Matos P, Ennis M, Turner S, Owen G, Bougueleret L, Xenarios I, Steinbeck C: Rhea-a manually curated resource of biochemical reactions. Nucleic Acids Res 2012, 40(Database issue):D754-D760.

33. Gene Ontology Consortium: The gene ontology project in $\mathbf{2 0 0 8 .}$ Nuc Acids Res 2008, 36:D440-D444.

34. The Universal Protein Resource (UniProt). Nuc Acids Res 2009 37(Database issue):D169-D174.

35. Webb EC: Enzyme Nomenclature, 1992: Recommendations of the Nomenclature Committee of the International Union of Biochemistry and Molecular Biology on the Nomenclature and Classification of Enzymes. New York: Academic Press; 1992.

36. Orth JD, Thiele I, Palsson BO: What is flux balance analysis? Nat Biotechnol 2010, 28(3):245-248.

37. MetaCyc Guide. [http://metacyc.org/MetaCycUserGuide.shtml]

38. Kanehisa M, Goto S, Sato Y, Furumichi M, Tanabe M: KEGG for integration and interpretation of large-scale molecular data sets. Nuc Acids Res 2012, 40(Database issue):D109-D114

39. Karp PD: Call for an enzyme genomics initiative. Genome Biol 2004 5:401.1-401.3

40. Pouliot $Y$, Karp PD: A survey of orphan enzyme activities. BMC Bioinformatics 2007, 8:244.

41. Rivals I, Personnaz L, Taing L, Potier MC: Enrichment or depletion of a GO category within a class of genes: Which test? Bioinformatics 2007 23:401-407.

42. Federhen S: The NCBI Taxonomy database. Nucleic Acids Res 2012 40(Database issue):D136-D143.

43. Weininger D: SMILES, a chemical language and information system. 1. Introduction to methodology and encoding rules. $J$ Chem Inf Comput Sci 1988, 28:31-36.

44. Arnaud M, Krieger C, Paley S, Karp PD: Curator's guide to pathway/genome databases. 2005. [http://brg.ai.sri.com/ptools/ curatorsguide.pdf]

45. Green ML, Karp PD: The outcomes of pathway database computations depend on pathway ontology. Nuc Acids Res 2006, 34:3687-3697.

46. Abubucker S, Segata N, Goll J, Schubert AM, Izard J, Cantarel BL, Rodriguez-Mueller B, Zucker J, Thiagarajan M, Henrissat B, White O, Kelley ST, Methe B, Schloss PD, Gevers D, Mitreva M, Huttenhower C: Metabolic reconstruction for metagenomic data and its application to the human microbiome. PLoS Comput Biol 2012, 8:1-17

47. Karp PD, Latendresse M, Caspi R: The Pathway Tools pathway prediction algorithm. Stand Genomic Sci 2011, 5(3):424-429.

doi:10.1186/1471-2105-14-112

Cite this article as: Altman et al:: A systematic comparison of the MetaCyc and KEGG pathway databases. BMC Bioinformatics 2013 14:112.

\section{Submit your next manuscript to BioMed Central and take full advantage of:}

- Convenient online submission

- Thorough peer review

- No space constraints or color figure charges

- Immediate publication on acceptance

- Inclusion in PubMed, CAS, Scopus and Google Scholar

- Research which is freely available for redistribution 